\title{
Quantization and diagnosis of Shanghuo (Heatiness) in Chinese medicine using a diagnostic scoring scheme and salivary biochemical parameters
}

Sijun Liu', Zhaosheng Huang ${ }^{1 *}$, Qingguang Wu', Zhangjie Huang ${ }^{1}$, Lirong Wu', Wenli Yan', David Lungpao Chang ${ }^{2}$, Zheng Yang ${ }^{2}$ and Zongwei Wang ${ }^{3^{*}}$

\begin{abstract}
Background: This study aims to establish a diagnostic scoring scheme for Shanghuo (Heatiness) and to evaluate whether Shanghuo is associated with biochemical parameters of salivary lysozyme (LYZ), salivary secreted immunoglobulin (S-IgA), salivary amylase (AMS), and saliva flow rate (SFR).

Methods: We collected 121 Shanghuo patients at the Affiliated Hospitals of Guangzhou University of Traditional Chinese Medicine in Guangdong Province, 60 cases as a Shanghuo recovered group, and 60 healthy cases as a healthy control group. The diagnostic scoring scheme was established by probability theory and maximum likelihood discriminatory analysis on the basis of epidemiology with the design of self-controlled clinical trial. Subsequently, we used the same methods to collect 120 Shanghuo patients, 60 Shanghuo recovered cases, and 60 healthy cases in both Hunan Province and Henan Province. The levels of LYZ, S-IgA, AMS, and SFR were tested when the patients suffered from Shanghuo or recovered, respectively.

Results: The diagnostic score table for Shanghuo syndrome was established first. In the retrospective tests, the sensitivity, specificity, accuracy, and positive likelihood ratio of the diagnostic score table were $98.9 \%, 93.5 \%, 97.5 \%$, and 14.34\%, respectively. In the prospective tests, the corresponding values were $94.9 \%, 85.7 \%, 91.7 \%$, and $6.64 \%$, respectively. Shanghuo was classified into three degrees based on the diagnostic scores, common Shanghuo: 63-120; serious Shanghuo: 121-150; very serious Shanghuo: $>150$. A negative correlation was found between Shanghuo and S-IgA $(R=-0.428 ; P=0.000)$. The level of S-lgA was also affected by seasonal and regional factors. No significant correlations were found between Shanghuo and the levels of LYZ, AMS, and SFR.
\end{abstract}

Conclusions: In this study, Shanghuo could be diagnosed by the combination of the diagnostic score table and S-IgA level.

\section{Background}

Huo (fire) is one of the five basic elements in traditional Chinese Medicine (TCM), together with $M u$ (wood), $T u$ (earth), Jin (metal), and Shui (water). Huo symbolizes active, positive, and warm substances or states [1]. When Huo becomes hyperactive in the body, this kind of internal

\footnotetext{
*Correspondence: vip.hzs@gzhtcm.edu.cn; zwang0@partners.org

'Guangzhou University of Traditional Chinese Medicine, 12 Jichang Road,

Guangzhou 510405, China

${ }^{3}$ Massachusetts General Hospital, Harvard Medical School, 55 Fruit St., Warren 317, Boston, MA 02114, USA

Full list of author information is available at the end of the article
}

Huo or internal $R e$ (heat) is idiomatically expressed as "Shanghuo" (Heatiness) [2].

Shanghuo in traditional Chinese medicine (TCM) is characterized by "redness, swelling, fever, and pain", usually emerges in the middle to late stages of infectious diseases, and also occurs in non-infectious diseases such as autoimmune diseases and endocrine diseases [3]. Typically, TCM physicians diagnose Shanghuo through inspection (visual examination), audio-olfactory examination, inquiry, and palpation, which are usually based on the

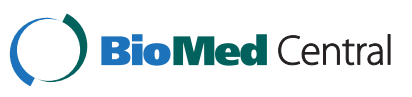

(c) 2014 Liu et al.; licensee BioMed Central Ltd. This is an open access article distributed under the terms of the Creative Commons Attribution License (http://creativecommons.org/licenses/by/2.0), which permits unrestricted use, distribution, and reproduction in any medium, provided the original work is properly cited. 
clinicians' subjective judgment, not quantified objective diagnostic parameters.

Human saliva is composed of $99.5 \%$ water, and the remaining $0.5 \%$ comprises electrolytes, mucus, glycoproteins, enzymes, and antibacterial compounds such as secretory IgA (S-IgA) and lysozyme (LYZ) [4-6]. S-IgA and LYZ protect the tooth enamel and prevent tooth decay and gum disease $[7,8]$. Studies have shown that some of the components in saliva can be used as biomarkers for diagnosis. Real-time PCR assays of both liquid and dried saliva specimens showed high sensitivity and specificity for detecting cytomegalovirus infection, suggesting their potential as screening tools for cytomegalovirus infection in newborns [9]. Zerr et al. [10] developed a noninvasive method for testing seriallycollected saliva specimens for human herpesvirus-6, and applied this method prospectively in children from birth to 2 years of age to determine the pattern of acquisition and natural history of human herpesvirus- 6 infection. More importantly, the easy access to saliva samples provides superiority for good repeatability and improved diagnostic accuracy.

In the oral immune system, S-IgA has been recognized as a critical immune barrier for infection and allergy [11]. Studies have shown that S-IgA agglutinates and binds the microbes in the oral cavity, inhibits their adhesive capability, and prevents them from clinging to the surface of the oral tissues. In addition, S-IgA inhibits bacterial growth and proliferation by changing the growth features of bacteria, and inhibits viral duplication with the help of complement proteins and lysosomes. Studies have found that the levels of S-IgA are evidently lower in patients with recurrent aphthae, recalcitrant oral ulceration, and oral lichen planus than in healthy control subjects [12-15].

Previous studies suggested that LYZ and SFR are associated with the occurrence and development of common oral diseases $[16,17]$. In general, a low level of LYZ is often correlated with the occurrence of oral infections and inflammatory diseases, and the frequency and proneness for dental plaque and dental caries [16].

This study aims to include the biochemical parameters of salivary LYZ, salivary S-IgA, salivary amylase (AMS), and saliva flow rate (SFR) in the diagnosis of the clinical syndrome of Shanghuo. We investigated Shanghuo cases from Guangdong, Hunan, and Henan provinces in China by establishing a diagnostic score table and a frequency table for Shanghuo, and evaluated whether Shanghuo is associated with the levels of LYZ, AMS, SFR, and S-IgA.

\section{Methods}

This study followed the Standards for Reporting of Diagnostic Accuracy (STARD) [18]. And it was approved by the Ethical review board of The First Affiliated Hospital of Guangzhou University of Chinese Medicine, Ethical Committee of Hunan University of TCM, and Ethical Committee of Henan University of TCM. All candidates signed a written consent followed by a baseline interview. During the interview, the candidates were asked to fill out a questionnaire, and the collected data included candidate's name, age, gender, address, the degree situation of observed index, the collection location, the collection time and the diagnostic results.

\section{Inclusion criteria \\ Shanghuo group}

The subjects in the Shanghuo group, who signed a written consent that approved by the research ethical committee complied with the diagnostic standards described below [19] and were simultaneously diagnosed as suffering from Shanghuo by three independent doctors in our group. If there was any disagreement on the diagnostic results among the doctors, the subject was excluded. The standards were as follows: internal exuberant fire and heat, characterized by common symptoms such as fever, thirst, and preference for cold drinks, epigastric burning, flushed face and hot eyes, constipation, scanty yellow urine, reddened tongue with yellow and dry coating, rapid pulse or vigorous and rapid pulse.

\section{Shanghuo recovered group}

The subjects in the Shanghuo recovered group were diagnosed as suffering from Shanghuo at the beginning of the study and recovered after 1-2 weeks. These subjects were also identified by three independent doctors in our project group with complete agreement. The recovered criteria matched those applied to the healthy control group.

\section{Healthy control group}

The subjects in the healthy control group had no excess Huo (or Re) syndrome, Xu Re (deficiency heat) syndrome, Хи Нио (deficiency fire) syndrome, for instance, oral dryness, oral ulcers, bitter taste, halitosis, swollen gums with pain or bleeding, stuffy nose, nasal dryness, nosebleed, ocular itch, eye gum, tinnitus, throat dryness, sore throat, acne, vertigo, scurf, vexing heat, low fever, insomnia, irascibility, yellow urine, reddened tongue, yellow tongue coating, constipation, thin rapid pulse, rapid flooding pulse, and were simultaneously diagnosed as not suffering from Shanghuo by three independent doctors in our group.

\section{Exclusion criteria}

The exclusion criteria for three groups were as follows: patients with malignant tumor diseases, patients with hepatitis, tuberculosis or other infectious diseases, patients with mental disorders, patients with diseases 
affecting the procedure for collecting saliva or affecting the follow-up study, non-acceptance of providing informed consent or low compliance from the patient.

\section{Collection of clinical data}

Between June 2003 and June 2008, we collected 121 Shanghuo patients at the Affiliated Hospitals of Guangzhou University of Traditional Chinese Medicine in Guangdong Province, 60 cases as the Shanghuo recovered group, and 60 healthy control cases as well--- to establish the diagnostic score table for Shanghuo. The same standards were followed when we collected another 120 Shanghuo cases, 60 recovered cases, and 60 healthy cases from both Hunan Province and Henan Province (Table 1). As showed in Table 1A, there were 102 male and 139 female in Guangdong province, 113 male and 127 female in Hunan province, 105 male and 135 female in Henan province. By chi-square test, there was no significant difference between three geographical regions in gender $\left(P>0.05, X^{2}=1.162, P=0.559\right)$. Through the test of homogeneity of variance, age and weight of subjects in three geographical regions has homogeneity of variance. By One-way ANOVA test (LSD), no significant difference was found between two of three groups in the factor of age and weight $(P=0.126,0.197$ and 0.144 respectively, with the age of subjects in Guangdong $v s$. Hunan, Guangdong vs. Henan, and Hunan vs. Henan. $P=0.082,0.095$, and 0.104 respectively, with the weight of subjects in Guangdong vs. Hunan, Guangdong vs. Henan, and Hunan vs. Henan Table 1B). Patient information was further summarized as Figure 1.

The patients in the three groups were asked to fill out a questionnaire (Additional file 1). This questionnaire on symptoms and signs was established by comprehensively listing the frequency of occurrence of symptoms and signs of Shanghuo according to the literature, selecting the symptoms with their frequency of occurrence with ranking of top 30 as the indices for four diagnostic methods, and setting a dichotomous variable (Yes/No).

\section{Saliva collection and salivary biochemical parameters test Sampling procedure}

Saliva samples were collected from 9:00-11:00 a.m. and 2:00-4:00 p.m., respectively. After rinsing the mouth with clean water, the subjects sat quietly for 3 minutes keeping the saliva in their mouth naturally, and then spat the saliva into a vial (non-irritant saliva) per minute. Saliva was collected for 10 minutes and the SFR was calculated (shown in 4.2.1). If the total amount of saliva over 10 minutes was less than $3 \mathrm{~mL}$, the collection duration was prolonged until $3 \mathrm{~mL}$ of saliva had been collected, and the total time was recorded for calculation of the SFR. The collected samples were stored at $-20^{\circ} \mathrm{C}$ until analysis.

\section{Determination of SFR, AMS, LYZ and S-IgA \\ SFR}

The SFR $(\mathrm{mL} / \mathrm{min})$ was calculated as the total volume of saliva collected per collecting time (total volume of saliva divided by minutes required).

\section{AMS}

After freeze-thawing, the saliva samples were diluted 20 times in saline. The level of AMS was determined by a commercially available AMS kit (Salivary Amylase Kit; HUMAN GmbH Co., Germany; Catalogue No. H100) in accordance with the manufacturer's protocol.

\section{$L Y Z$}

The level of LYZ was examined using LYZ kits (Nanjing Jiancheng Bioengineering Research Institute, China; Catalogue No. 20060922, 20061025, and 20070412) in accordance with the producer's guidelines, by a 752

Table 1 The clinical data of subjects

\begin{tabular}{|c|c|c|c|c|c|c|c|c|c|c|}
\hline \multicolumn{11}{|c|}{ A. The number of subjects from three geographical regions } \\
\hline \multirow[t]{2}{*}{ Gender } & \multicolumn{3}{|c|}{$\begin{array}{l}\text { Number of subjects in Guangdong } \\
\text { province }\end{array}$} & \multicolumn{3}{|c|}{ Number of subjects in Hunan province } & \multicolumn{3}{|c|}{ Number of subjects in Henan province } & \multirow[t]{2}{*}{ Total } \\
\hline & Heatiness & Recovered & Healthy control & Heatiness & Recovered & Healthy control & Heatiness & Recovered & Healthy control & \\
\hline Male & 51 & 24 & 27 & 55 & 28 & 30 & 50 & 30 & 25 & 318 \\
\hline Female & 70 & 36 & 33 & 65 & 32 & 30 & 70 & 30 & 35 & 391 \\
\hline Total & 121 & 60 & 60 & 120 & 60 & 60 & 120 & 60 & 60 & 721 \\
\hline \multicolumn{11}{|c|}{ B. The situation of age and weight with subjects } \\
\hline \multicolumn{4}{|c|}{ Geographical regions } & \multicolumn{3}{|c|}{ Number of subjects $(\mathrm{N})$} & \multicolumn{2}{|l|}{ Age (year) } & \multicolumn{2}{|l|}{ Weight (kg.) } \\
\hline \multicolumn{4}{|c|}{ Subjects in Guangdong province } & \multicolumn{3}{|l|}{241} & \multicolumn{2}{|c|}{$32.415(15.220)$} & \multicolumn{2}{|l|}{$49.541(17.040)$} \\
\hline \multicolumn{4}{|c|}{ Subjects in Hunan province } & \multicolumn{3}{|l|}{240} & \multicolumn{2}{|c|}{$33.250(19.085)$} & \multicolumn{2}{|l|}{$52.440(15.115)$} \\
\hline \multicolumn{4}{|c|}{ Subjects in Henan province } & \multicolumn{3}{|l|}{240} & \multicolumn{2}{|c|}{$31.215(12.550)$} & \multicolumn{2}{|l|}{$54.755(13.205)$} \\
\hline
\end{tabular}

All data were presented as mean (SD). 


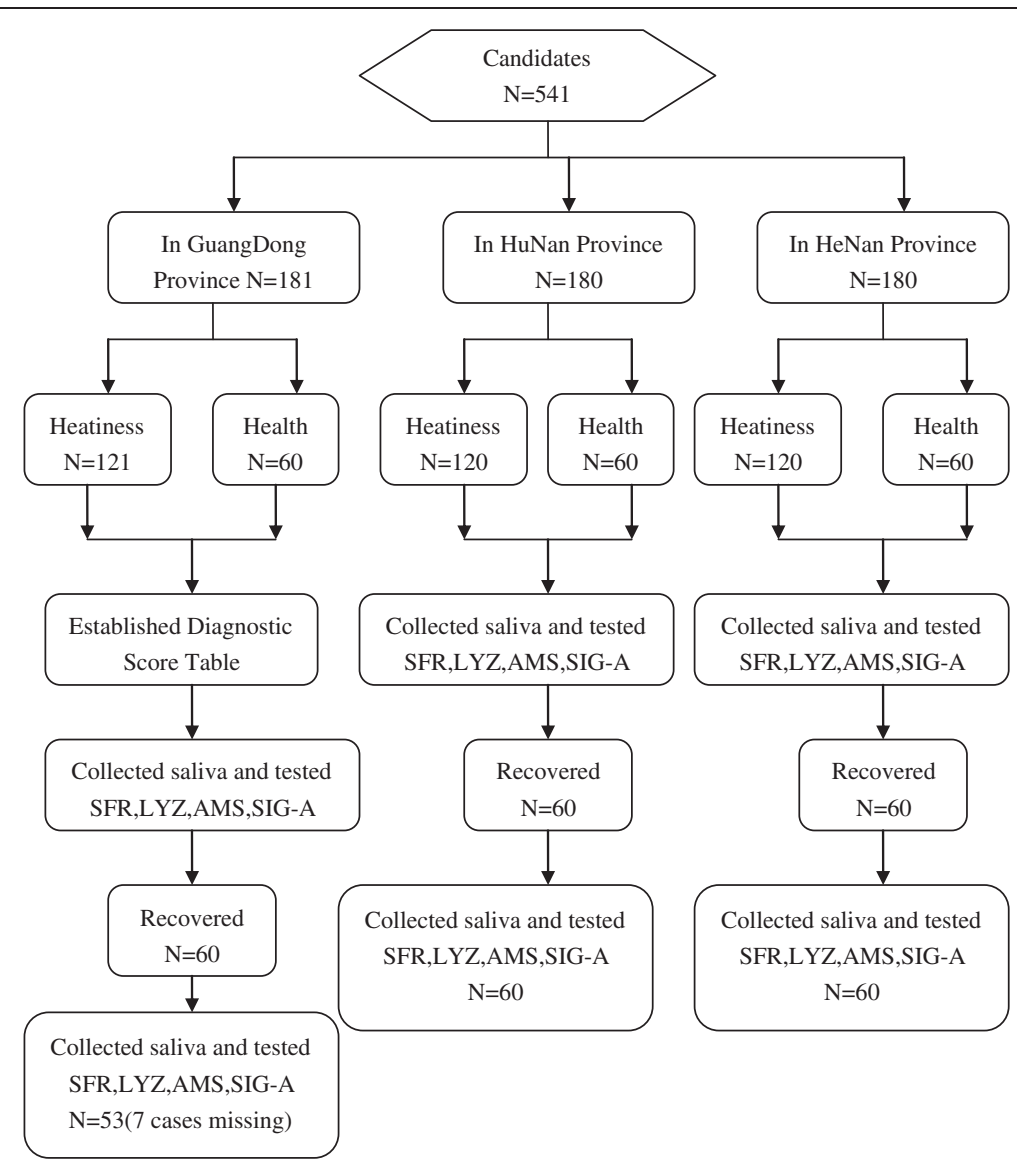

Figure 1 The flow diagram of the study.

Spectrophotometer (Shanghai Jingke Equipment Co., Ltd., China).

\section{$S-\lg A$}

S-IgA was determined with S-IgA Kits (Beijing North Institute of Biotechnology, China; Catalogue No. 061120, 061230, 070412, and 070420) by an SN-695B radioimmunoassay measuring instrument (Shanghai Hesuo Rihuan Photoelectric Instrument Co., Ltd., China).

\section{Statistical analysis}

The chi-square test was used to analyze the observed indices. A method for maximum likelihood discriminatory analysis was used to establish the diagnostic score table for Shanghuo [20]. The Wilcoxon rank sum correlation test was used to analyze the correlations between the Shanghuo scores and the levels of SFR, LYZ, AMS, or $S$-IgA. A paired sample $t$-test was used to study the seasonal and regional differences in S-IgA between the Shanghuo patients and healthy controls. All of the statistical tests were two-tailed. Values of $P<0.05$ were considered as statistical significance.

\section{Results}

\section{Diagnostic score tables}

We calculated the number of Shanghuo cases and healthy cases with the occurrence of each symptom index among the 30 indices on a one-by-one basis with the chi-square test, and excluded the indices with values of $P>0.05$.

Healthy negative means that the number of the index did not appear in the healthy control group, while healthy positive means the number of the index did appear in the group. Similarly, Shanghuo negative means that the number of the index did not appear in the Shanghuo group, while Shanghuo positive means the number of the index did appear in the group. Using the chi-square test, we found that (5) Constant hunger, (12) Red eyes, and (17) Sputum showed no significant differences between the healthy control and Shanghuo groups $(P=0.659$ for constant hunger, $P=0.150$ for red eyes and $P=0.117$ for Sputum). However, the remaining 27 indices did differ significantly between the healthy control and Shanghuo groups ( $P=0.004$ for Stuffy nose, $P=0.012$ for Nosebleed, $P=0.004$ for Scurf, $P=0.003$ for Reddened tongue, $P=0.002$ for Rapid flooding pulse, and $P=0.000$ 
for the other 22 indices). Therefore, these 27 indices were retained (Table 2).

Next, we calculated the exponential value of each index in the healthy control and Shanghuo groups by the conditional probability conversion formula [20]. The formula was:

$$
\begin{aligned}
L_{i j} & =\left[\lg \left(X_{j} / Y_{i}\right)+1\right] \times 10\left(X_{j} / Y_{i}>0\right) \text { and } L_{i j} \\
& =-10\left(X_{j} / Y_{i}-0\right),
\end{aligned}
$$

where $L_{i j}$ is the exponential value of the index, $X_{j}$ is the number of Shanghuo or healthy cases with the index, and $Y_{i}$ is the total number of cases collected.
By using the frequency of occurrence of each index, the exponential value of each index in the healthy cases was subtracted and the absolute value was adopted. Similarly, the exponential value of each index in the Shanghuo cases was subtracted and the absolute value was adopted. Subsequently, the two absolute values were summed up to calculate the score of each index. For example, for all 60 cases in the healthy group, (1) Ulcer did not appear. Thus, the negative conditional probability of ulcer was $100 \%$ and the positive conditional probability of ulcer was $0 \%$. According to the conditional probability conversion formula, the negative exponential value of ulcer in the healthy group was 10 and the positive exponential value was -10 . The negative exponential

\begin{tabular}{|c|c|c|c|c|c|c|}
\hline Indices & $\begin{array}{l}\text { Healthy } \\
\text { negative }\end{array}$ & $\begin{array}{l}\text { Healthy } \\
\text { positive }\end{array}$ & $\begin{array}{l}\text { Heatiness } \\
\text { negative }\end{array}$ & $\begin{array}{l}\text { Heatiness } \\
\text { positive }\end{array}$ & $\begin{array}{l}\text { Chi-square } \\
\text { value }\end{array}$ & $P$ \\
\hline (1)Ulcer & 60 & 0 & 39 & 82 & 74.340 & 0.000 \\
\hline (2)Oral dryness & 50 & 10 & 20 & 101 & 75.475 & 0.000 \\
\hline (3)Bitter taste & 59 & 1 & 84 & 37 & 20.214 & 0.000 \\
\hline (4)Halitosis & 60 & 0 & 81 & 40 & 25.462 & 0.000 \\
\hline (5)Constant hungering & 45 & 15 & 87 & 34 & 0.195 & 0.659 \\
\hline $\begin{array}{l}\text { (6)Swollen gums with pain } \\
\text { or bleeding }\end{array}$ & 55 & 5 & 23 & 98 & 86.349 & 0.000 \\
\hline (7)Stuffy nose & 55 & 5 & 89 & 32 & 8.092 & 0.004 \\
\hline (8)Nasal dryness & 58 & 2 & 57 & 64 & 42.523 & 0.000 \\
\hline (9)Nosebleed & 60 & 0 & 109 & 12 & 6.373 & 0.012 \\
\hline (10)Ocular dryness & 50 & 10 & 46 & 75 & 33.071 & 0.000 \\
\hline (11)Ocular itch & 57 & 3 & 87 & 34 & 13.160 & 0.000 \\
\hline (12)Red eyes & 59 & 1 & 113 & 8 & 2.076 & 0.150 \\
\hline (13)Eye gum & 59 & 1 & 66 & 55 & 35.994 & 0.000 \\
\hline (14)Tinnitus & 59 & 1 & 101 & 20 & 20.984 & 0.000 \\
\hline (15)Throat dryness & 57 & 3 & 56 & 65 & 40.591 & 0.000 \\
\hline (16)Sore throat & 59 & 1 & 83 & 38 & 20.984 & 0.000 \\
\hline (17)Sputum & 57 & 3 & 106 & 15 & 2.450 & 0.117 \\
\hline (18)Acne & 48 & 12 & 55 & 66 & 19.519 & 0.000 \\
\hline (19)Vertigo & 55 & 5 & 90 & 31 & 7.522 & 0.006 \\
\hline (20)Scurf & 55 & 5 & 89 & 32 & 8.092 & 0.004 \\
\hline (21)Vexing heat & 60 & 0 & 83 & 38 & 23.85 & 0.000 \\
\hline (22)Low fever & 60 & 0 & 89 & 32 & 19.276 & 0.000 \\
\hline (23)Insomnia & 53 & 7 & 57 & 64 & 28.596 & 0.000 \\
\hline (24)Irascibility & 59 & 1 & 67 & 54 & 34.998 & 0.000 \\
\hline (25)Yellow urine & 55 & 5 & 72 & 49 & 19.821 & 0.000 \\
\hline (26)Constipation & 53 & 7 & 61 & 60 & 24.739 & 0.000 \\
\hline (27)Reddened tongue & 58 & 2 & 97 & 24 & 8.879 & 0.003 \\
\hline (28)Yellow tongue coating & 59 & 1 & 83 & 38 & 20.984 & 0.000 \\
\hline (29)Thin rapid pulse & 55 & 5 & 62 & 59 & 28.681 & 0.000 \\
\hline (30)Rapid flooding pulse & 60 & 0 & 104 & 17 & 9.304 & 0.002 \\
\hline
\end{tabular}

Table 2 The result of chi-square test 
value of ulcer in the Shanghuo group was 5 and the positive exponential value was 8 . Therefore, the final score for ulcer was $|10-(-10)|+|5-8|=23$ (Additional file 2).

After simplification of the above table, we obtained a diagnostic score table for Shanghuo (Table 3).

\section{Diagnostic threshold values}

At the beginning of diagnosis, each index in Table 2 was assumed "negative", and the exponential values of the 27 "negative" indices of Shanghuo in Table 3 were summed to obtain the exponential value sum for Shanghuo, i.e., 202. The exponential value sum for non-Shanghuo obtained in a similar way was 265 , and the relative exponential sum of value for Shanghuo was 63. In other words, greater than 63 of the total score for "positive" indices is the diagnostic threshold value for Shanghuo.

\section{Clinical application}

The test for normality of the total cases collected in Guangzhou, Henan, and Hunan provinces in reference to the diagnostic score table showed that the score values for severity of the Shanghuo cases were consistent with a normal distribution (Figure 2). Statistically, if a digital index is consistent with a normal distribution, the percentage grading will be applicable.

According to the method for percentiles $(p): P_{33.3}=120.63 \approx$ $120 ; P_{66.7}=149.37 \approx 150$, Shanghuo could be classified into three degrees based on the diagnostic scores: common Shanghuo: 63-120; serious Shanghuo: 121-150; very serious Shanghuo: $>150$.

\section{Diagnostic tests}

\section{Retrospective tests}

By virtue of the established diagnostic score table for Shanghuo, we first diagnosed the 361 cases (including all Shanghuo cases in the three regions) in conformity with the Shanghuo diagnostic standards, and calculated that the sensitivity, specificity, accuracy, and positive likelihood ratio were $98.9 \%, 93.5 \%, 97.5 \%$, and $14.34 \%$, respectively.

\section{Prospective tests}

We diagnosed the 180 cases (including all healthy cases in the three regions) by virtue of the established diagnostic score table for Shanghuo, and found that the sensitivity, specificity, accuracy, and positive likelihood ratio of the test were $94.9 \%, 85.7 \%, 91.7 \%$, and $6.64 \%$, respectively.

\section{Assessment of diagnostic efficiency with a ROC curve}

We assessed the cases collected from the three geographical regions by the diagnostic score table. Taking the sensitivity as the ordinate axis and the specificity as the abscissa axis, we obtained a receiver operating characteristic (ROC) curve to appraise the diagnostic efficiency by calculating the area under the ROC curve (Figure 3). The size of the integrated area under the curve was closely related to the reliability of a diagnostic trial.

\section{Analysis of comparisons between Shanghuo and S-IgA Comparisons between the Shanghuo group and Shanghuo recovered group for salivary biochemical parameters}

The SFR values were $0.430 \pm 0.290 \mathrm{~mL} / \mathrm{min}$ and $0.450 \pm$ $0.230 \mathrm{~mL} / \mathrm{min}$ before and after Shanghuo recovery, respectively $(P=0.503)$. The levels of LYZ and AMS did not show any significant differences before and after Shanghuo recovery $(P=0.300$ and $P=0.240$, respectively). However, the level of S-IGA was significantly elevated from $439.770 \pm 60.710 \mathrm{mg} / \mathrm{dL}$ with Shanghuo to $1348.570 \pm 170.690 \mathrm{mg} / \mathrm{dL}$ after recovery $(P<0.001)$ (Table 4).

\section{Correlations between Shanghuo and the levels of SFR, LYZ, AMS, and S-IgA}

The statistical analyses of the Shanghuo scores and the correlations between Shanghuo and the levels of SFR, LYZ, AMS, and S-IgA were performed by the Wilcoxon rank sum correlation test, using the corresponding index of the same patient. The data suggested that there was a significantly negative correlation between the diagnostic score for Shanghuo and S-IgA $(R=-0.428 ; P=0.000)$,

Table 3 Diagnostic score table of heatiness

\begin{tabular}{|c|c|c|c|c|c|}
\hline Indices & Value & Indices & Value & Indices & Value \\
\hline (1)Ulcer & 23 & (10)Eye itching & 8 & (19)Low-grade fever & 15 \\
\hline (2)Dry mouth & 14 & (11)Secretion of the eyes & 18 & (20)Insomnia & 8 \\
\hline (3)Bitterness in the mouth & 15 & (12)Tinnitus & 11 & (21)Tantrum & 17 \\
\hline (4)Bad breath & 17 & (13)Pharyngoxerosis & 13 & (22)Yellow urine & 9 \\
\hline (5)Gum swelling and aching or bleeding & 17 & (14)Sore throat & 15 & (23)Constipation & 8 \\
\hline (6)Nasal obstruction & 6 & (15)Acne & 6 & (24)Red tongue & 9 \\
\hline (7)Nasal dryness & 15 & (16)Dizziness & 6 & (25)Yellow coating & 15 \\
\hline (8)Nose bleeding & 10 & (17)Scurf desquamation & 6 & (26)Frequent and weak pulse & 11 \\
\hline (9)Dryness of the eyes & 9 & (18)Dryness-heat & 17 & (27)Frequent and strong pulse & 13 \\
\hline
\end{tabular}




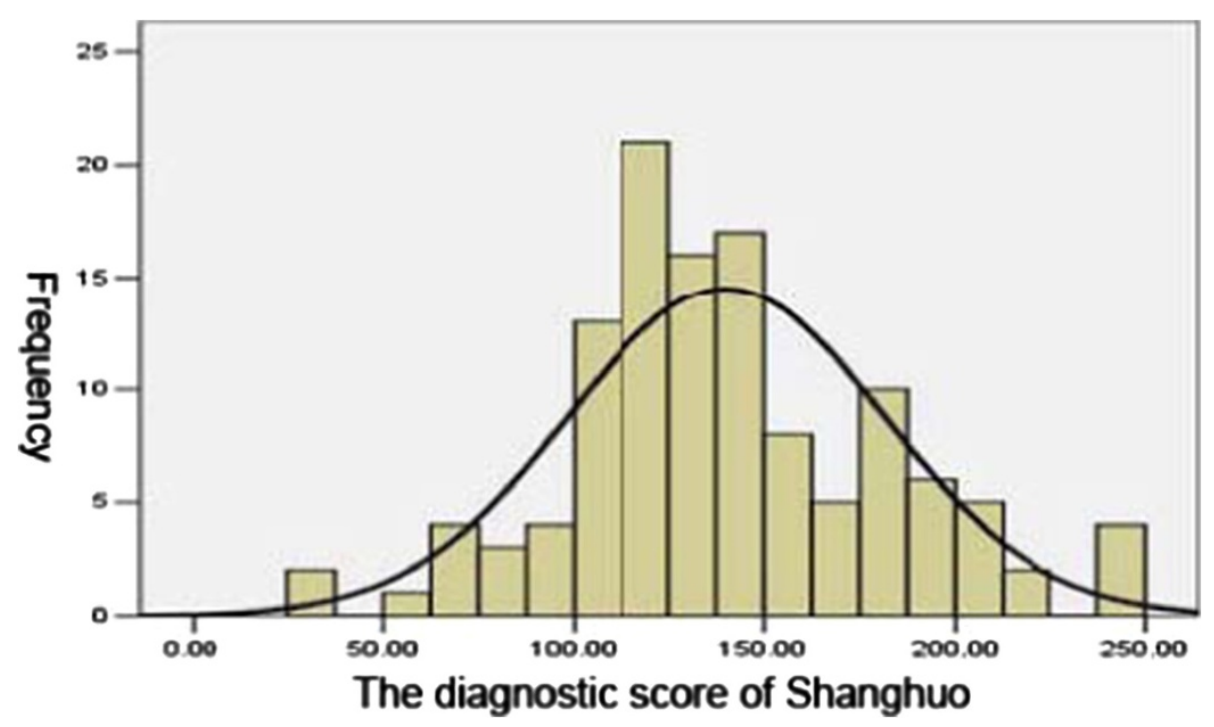

Figure 2 The normal distribution test.

but no significant correlation between the other indices and the diagnostic score for Shanghuo (Table 5).

\section{Factors associated with S-IgA acquisition}

To examine whether S-IgA had seasonal effects on Shanghuo, we collected and evaluated 361 Shanghuo cases collected from above three regions (121 cases in Guangdong Province, 120 cases in Hunan Province, and 120 cases in Henan Province) for seasonal divisions on the basis of the 24 solar terms in the lunar calendar (Table 6). The results demonstrated that there were significant differences between spring and summer $(P=0.000)$, spring and autumn $(P=0.000)$, spring and winter $(P=0.000)$, summer and winter $(P=0.047)$, summer and autumn $(P=0.000)$, and autumn and winter $(P=0.000)$ (Table 6A). The different levels of S-IgA in different seasons suggested that the seasonal factor might be considered when studying Shanghuo and S-IgA.

Through comparisons among groups of cases from different regions, we found that there were significant differences between Guangzhou and Henan $(P=0.000)$ and Guangzhou and Hunan $(P=0.000)$, but no significant difference between Hunan and Henan $(P=0.116)$ (Table 6B). These data suggested that the levels of S-IgA might be affected by region.

\section{Evaluation of Shanghuo by combination of the diagnostic} score tables and S-IgA levels

Finally, we investigated the consistency of Shanghuo cases collected from Guangzhou, Henan, and Hunan

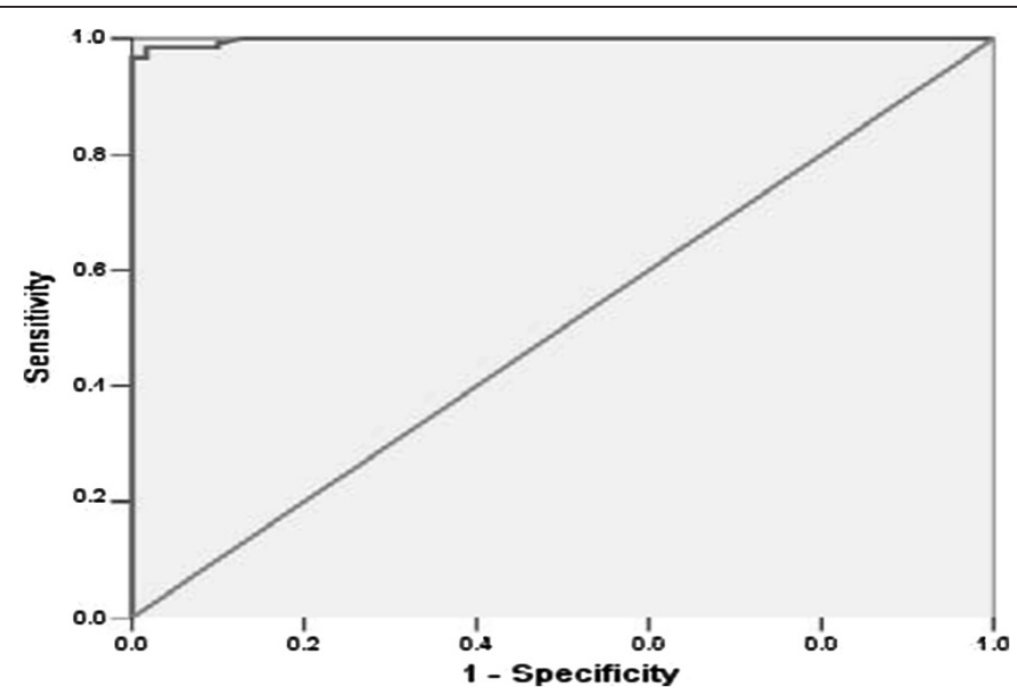

Figure 3 Assessment of diagnostic efficiency with a ROC curve. 
Table 4 Self-paired comparison of the parameter changes

\begin{tabular}{llllll}
\hline Index & Status: & $\mathbf{N}$ & Value & $\mathbf{T}$ & $\boldsymbol{P}$ \\
\hline SFR & Heatiness & 60 & $0.430(0.290)$ & -0.673 & 0.503 \\
& Recovered & 60 & $0.450(0.230)$ & & \\
LYZ & Heatiness & 60 & $33.420(5.830)$ & 3.087 & $0.003^{* *}$ \\
& Recovered & 60 & $34.250(5.200)$ & & \\
AMS & Heatiness & 60 & $112916.000(83073.000)$ & 2.315 & $0.024^{*}$ \\
& Recovered & 60 & $87309.000(86224.000)$ & & \\
S-IgA & Heatiness & 60 & $439.770(607.100)$ & -4.530 & $0.000^{* * *}$ \\
& Recovered & 60 & $1348.570(1706.900)$ & & \\
\hline
\end{tabular}

All data were presented as mean (SD). ${ }^{*} P<0.05,{ }^{* *} P<0.01,{ }^{* *} P<0.001$, by paired $t$ test.

provinces before and after the experiment, using the S-IgA level and diagnostic score table from Guangzhou. After the clinical diagnosis was executed by three clinicians individually, the $\mathrm{D}$ values were compared with the diagnostic score table, and the S-IgA levels without a quantitative threshold value were compared with the trend. The results showed that for diagnosis with the $\mathrm{D}$ value independently, the consistency with the clinical diagnosis of Shanghuo was $89.6 \%$. When evaluating S-IgA independently, the consistency with the clinical diagnosis of Shanghuo was $48.2 \%$. For combined evaluation with D value and S-IgA, the consistency with the clinical diagnosis of Shanghuo was 92.2\%. These data indicated that evaluation by combining $\mathrm{D}$ value and S-IgA increased the diagnostic efficiency for Shanghuo.

\section{Discussion}

As one essential element in TCM, huo provides energy and power to human body. Shanghuo is a state that huo becomes hyperactive and aggressive. The oral ailments associated with Shanghuo syndrome include oral dryness, mucosal ulcer, and gum bleeding, swelling, and pain. These symptoms are similar to those of Sjogren syndrome, recurrent aphthae, gingivitis, periodontitis, and caries [21].

The production of saliva is affected by many factors, such as temperature, food, mood, and different times of the day [4]. To make saliva samples more repeatable, we developed a self-controlled experiment and collected

Table 5 Correlation of the diagnostic score of Shanghuo with the saliva biochemical parameters

\begin{tabular}{llll}
\hline & $\mathbf{N}$ & $\mathbf{P}$ & $\mathbf{R}$ \\
\hline Score and SFR & 174 & 0.973 & -0.627 \\
Score and LYZ & 174 & 0.592 & 0.224 \\
Score and AMS & 174 & 0.121 & 0.365 \\
Score and S-IgA & 174 & $0.000^{* * *}$ & -0.428 \\
\hline
\end{tabular}

Data from Guangzhou province, included 121 heatiness cases and 60 healthy cases, excluded 7 cases whose saliva biochemical parameters were not available. ${ }^{* * *} P<0.001$, by paired $t$ test.
Table 6 Seasonal and regional comparison of S-IgA

\begin{tabular}{lcl}
\hline A: Seasonal comparison of S-IgA & \\
\hline Season & $\mathbf{N}$ & Value of S-IgA \\
\hline Spring & 89 & $647.177(159.324)^{*}$ \\
Summer & 92 & $478.598(138.558)^{*}$ \\
Autumn & 84 & $861.726(75.147)^{*}$ \\
Winter & 96 & $513.573(86.463)^{*}$ \\
B: Regional comparison of S-IgA & \\
Regions & $\mathbf{N}$ & Value of S-IgA \\
Guangdong & 121 & $604.891(171.961)^{\#}$ \\
Hunan & 120 & $720.917(207.122)$ \\
Henan & 120 & $697.73(188.096)$
\end{tabular}

All data were presented as mean (SD). ${ }^{*} P<0.01$, vs. any other seasons; ${ }^{\#} P<$ 0.01 , vs. any other provinces.

saliva in the same time windows, i.e., 9:00-11:00 a.m. and 2:00-4:00 p.m.

In this study, we found that the level of S-IgA was significantly decreased in the Shanghuo group compared with the healthy control group, suggesting that S-IgA might represent a diagnosic marker for Shanghuo. Further analyses indicated that the level of S-IgA was negatively correlated with the severity of Shanghuo. The levels of S-IgA in Shanghuo patients varied among different seasons and regions, suggesting that the influences of seasons and regions should be considered when S-IgA was used as a marker for Shanghuo.

According to our clinical observations, many Shanghuo patients had xerostomia, albeit to different extents. As the first study on the correlation between xerostomia, SFR, and Shanghuo, we investigated whether the SFR in patients was associated with Shanghuo. However, our data did not show any direct evidence for this hypothesis. Further studies need to be undertaken to address this issue.

Quantitative diagnosis is an interdisciplinary subject and is the probability theory of statistics for the treatment effects evaluation and prognostic diagnosis [22]. Shanghuo involves many viscera, with different manifestations in different viscera, and there have been no clear definitions for the essence and diagnostic standards of Shanghuo. Here we established a diagnostic scoring scheme with the methods of probability theory and maximum likelihood discriminatory analysis on the basis of epidemiology.

From the diagnostic score table and S-IgA before and after Shanghuo and the consistency with the clinical diagnosis, we found that the combination of the diagnostic score table and S-IgA could increase the diagnostic efficiency. The diagnostic score table is intuitive and capable of direct diagnosis, albeit through 27 diagnostic indices of TCM, if tongue coating and pulse are diagnosed by modern machines [23]. We established the 
diagnostic score table by statistical methods and made an attempt for the diagnosis of Shanghuo quantitative research. In addition, we used modern machines for tongue coating and pulse, applied the diagnostic score table, and found that the scores for patients with Shanghuo could be calculated to indicate the severity of the Shanghuo, and that these scores could be used to evaluate the effectiveness of curative treatments.

In this study, we found that the levels of LYZ, AMS, and SFR had no significant correlations with Shanghuo, while S-IgA was associated with Shanghuo even the S-lgA level might be varied by different regions and seasons. We believe that quantification using the diagnostic score table and the level of S-IgA will improve the diagnostic accuracy and efficiency of Shanghuo.

Still, some limitations of this work should be considered. First, participants were not randomly selected and were limited to the three particular geographic regions in China. And the study was generally characterized by small sample sizes and may not be representative of the population. Secondly, the collection of data was objective because of lacking gold standard for Shanghuo, even though the recruited candidates simultaneously diagnosed by three independent doctors. These design and sampling strategies may reduce the external validity of our study. Also, because of the situation of pulse was included in the diagnostic score table, when this diagnostic scoring scheme was applied in clinic, the theory of pulse in TCM should be known. These limitations need to be considered when our findings are interpreted.

\section{Conclusions}

This study demonstrated that Shanghuo could be diagnosed by a combination of diagnostic score tables and S-lgA levels.

\section{Additional files}

Additional file 1: The representative questionnaire used for heatiness and control subjects.

Additional file 2: The indexs and values of heatiness symptom.

\section{Abbreviations}

LYZ: Salivary lysozyme; S-IgA: Salivary secreted immunoglobulin; AMS: Salivary amylase; SFR: Saliva flow rate; TCM: Traditional Chinese Medicine.

\section{Competing interests}

The authors declare that they have no competing interests.

\section{Authors' contributions}

ZH, SL, QW, DC, ZY and ZW conceived and designed the study. SL performed the experiment. SL, ZH, LW and WY performed the statistical analysis. SL and ZW wrote the manuscript. All authors read and approved the final manuscript.

\section{Acknowledgements}

This study was supported jointly by a grant from the National Natural Science Foundation of China (No. 81273817) and the program of
Guangdong Province administration of traditional Chinese medicine (No. 20121225).The authors are also grateful to Colgate-Palmolive (China) Company Ltd. for funding this study.

\section{Author details}

'Guangzhou University of Traditional Chinese Medicine, 12 Jichang Road, Guangzhou 510405, China. ${ }^{2}$ Colgate-Palmolive (China) Co. Ltd., Guangzhou, China. ${ }^{3}$ Massachusetts General Hospital, Harvard Medical School, 55 Fruit St., Warren 317, Boston, MA 02114, USA.

Received: 9 April 2013 Accepted: 31 December 2013

Published: 4 January 2014

\section{References}

1. Sun GR: Basic theory of traditional Chinese Medicine. Beijing: China Press of traditional Chinese Medicine; 2008.

2. Li JW, Deng TT: Dictionary of traditional Chinese Medicine. Beijing: People's Medical Publishing; 1995:942.

3. Guan SX, Chen JF, Ma YL: Clinical study on the relationship between HUO-RE (fire-heat) syndrome and plasma immune cell factor level changes at initial condition of acute cerebral infarction. Fang She Mian Yi Za Zhi 2000, 13:331-333.

4. Llena-Puy C: The role of saliva in maintaining oral health and as an aid to diagnosis. Med Oral Patol Oral Cir Bucal 2006, 11(5):E449-E455.

5. Toto PD: A look at saliva: the salivary antibacterial proteins. Bur 1985, 87(2):33-34.

6. Jankowska AK, Waszkiel D, Kobus A, Zwierz K: Saliva as a main component of oral cavity ecosystem. Part II. Defense mechanisms. Wiad Lek 2007, 60(5-6):253-257.

7. Deng HS: Determination and analysis of lysozyme and IgA in dental plaque and saliva and IgG in saliva of patients with dental caries and gingivitis. Zhonghua Yi Xue Za Zhi 1984, 64(10):598-600.

8. Siamopoulou-Mavridou A, Mavridis A, Galanakis E, Vasakos S, Fatourou H, Lapatsanis P: Flow rate and chemistry of parotid saliva related to dental caries and gingivitis in patients with thalassaemia major. Int J Paediatr Dent 1992, 2(2):93-97.

9. Boppana SB, Ross SA, Shimamura M, Palmer AL, Ahmed A, Michaels MG Sánchez PJ, Bernstein DI, Tolan RW Jr, Novak Z, Chowdhury N, Britt WJ, Fowler KB: Saliva polymerase-chain-reaction assay for cytomegalovirus screening in newborns. N Engl J Med 2011, 364(22):2111-2118.

10. Zerr DM, Meier AS, Selke SS, Frenkel LM, Huang M-L, Wald A, Rhoads MP, Nguy L, Bornemann R, Morrow RA, et al: A population-based study of primary human herpesvirus 6 infection. N Engl J Med 2005, 352(8):768-776.

11. Mestecky J, Russell MW: Specific antibody activity, glycan heterogeneity and polyreactivity contribute to the protective activity of S-IgA at mucosal surfaces. Immunol Lett 2009, 124:57-62.

12. Wu HH, Wang YQ: Observation of changes of saliva and serum Immumoglobulins in recurrent aphthae Patients. Lin Chuang Kou Qiang Yi Xue Za Zhi 2005, 21(5):308-309.

13. Li R, Zhang HY, Zhang JP, Hao ST: Stubborn oral ulcer in immune status inquiry. Xian Dai Kou Qiang Yi Xue Za Zhi 1996, 10(4):245.

14. Wei SG, Luo FZ, Zhang LD, Y.T.: Recurrent respiratory tract infection in serum VA and SlgA in saliva. Ji Chu Yi Xue Yu Lin Chuang 1994, 14:65,69.

15. He XN, Bao JR, Wang YM: Preliminary study of immunoblobulion and oral lichenplanus. NingXia Yi Xue Za Zhi 1999, 21:274-275.

16. Han JX: Salivary lysozyme and common oral disease. Guo Ji Kou Qiang Yi Xue Za Zhi 2006, 33:284-285.

17. Altarawneh S, Bencharit S, Mendoza L, Curran A, Barrow D, Barros S, Preisser J, Loewy ZG, Gendreau L, Offenbacher S: Clinical and histological findings of denture stomatitis as related to intraoral colonization patterns of candida albicans, salivary flow, and dry mouth. J Prosthodont 2013, 22(1):13-22.

18. Bossuyt PM, Reitsma JB, Bruns DE, Gatsonis CA, Glasziou PP, Irwig LM, Moher D, Rennie D, de Vet HC, Lijmer JG: The STARD statement for reporting studies of diagnostic accuracy: explanation and elaboration. Clin Chem 2003, 49(1):7-18.

19. General Administration of Quality Supervision: The people's Republic of China national standards of TCM clinical diagnosis and treatment in terms of symptom part. Beijing: Standards Press of China; 1997.

20. Luo TL, Chen GL, Zhao YQ, Chen ZQ, Shi L, Zhao JF: Quantitative differential diagnosis and clinical assessment of five kinds of Chinese medicine liver disease. Zhong Guo Xian Dai Yi Xue Za Zhi 1999, 9:29-30. 
21. Yan ZM, Wei P, Qiang L: Clinical assessment of oral diagnostic items in 2002 classification criteria for primary Sjgren's syndrome. Bei Jing Da Xue Xue Bao (Health Sciences) 2012, 44:51-54.

22. Jiang J: Chinese medicine symptom quantization diagnostic criteria research method general situation. Zhong Guo Yi Xue Za Zhi 2010, 25:94-96.

23. Hu J, Zhao T, Xu G, Wu L, Kou Q, Zhuang H, Li Z, Su M, Zhang G, et al: Assessment on the accuracy of four types of pulse by SM-1A TCM pulse detector. Shi Jie Ke Xue Ji Shu Za Zhi 2011, 13(1):74-77.

doi:10.1186/1749-8546-9-2

Cite this article as: Liu et al:: Quantization and diagnosis of Shanghuo

(Heatiness) in Chinese medicine using a diagnostic scoring scheme and

salivary biochemical parameters. Chinese Medicine 2014 9:2.

\section{Submit your next manuscript to BioMed Central and take full advantage of:}

- Convenient online submission

- Thorough peer review

- No space constraints or color figure charges

- Immediate publication on acceptance

- Inclusion in PubMed, CAS, Scopus and Google Scholar

- Research which is freely available for redistribution 